\title{
Vibrational energy harvesting device with magnetic tip mass
}

\author{
Fedor Fedulov*, Leonid Fetisov \\ Moscow Technological University (MIREA), Research-Education Center "Magnetoelectric materials \\ and devices", 78 Vernadsky Avenue, Moscow, 119454, Russia
}

\begin{abstract}
In this paper, the energy-harvesting device based on vibrational piezoelectric transducer for $\mathrm{AC}$ magnetic field conversion is presented. The harvester consists of the transducer and an AC/DC-converter. The load dependencies of the transducer showed that the maximal rms power reached a peak of $P_{r m s}=37 \mu \mathrm{W}$ at $f=50 \mathrm{~Hz}$ and $P_{r m s}=200 \mu \mathrm{W}$ at $f=48 \mathrm{~Hz}$. If the harvester is placed into an external AC magnetic field $h(t)$ of the frequency $f=48 \mathrm{~Hz}$ and constant amplitude of $h=5 \mathrm{Oe}$, DC voltage of fixed level (1.8 $\mathrm{V}, 2.5 \mathrm{~V}, 3.3 \mathrm{~V}$ or $3.6 \mathrm{~V}$ ) is available on the output. Charging time depends on the voltage level and has a maximum value of $t \approx 11 \mathrm{~s}$ for $3.6 \mathrm{~V}$ and minimum value $t \approx 6 \mathrm{~s}$ for $1.8 \mathrm{~V}$.
\end{abstract}

\section{Introduction}

Energy harvesting (EH) technology is one of the fast-growing areas of scientific investigations. The main idea of $\mathrm{EH}$ techniques is conversion of widespread solar, wind, thermal, mechanical and AC magnetic field energy into electrical one [1]. The stored electrical energy is used for supplying low-power electronics. Solar and wind energy are the most common energy sources. However, thermal, mechanical and AC magnetic field energy sources are the promising for industrial applications. This is a reason why effective mechanical and magnetic energy conversion is paid a great attention of scientists and engineers.

The main sources of mechanical vibrations in urban settings are as follows: all kinds of public transport (cars, buses, trams, trolleys, subway and railway transport) and its infrastructure (motorways and railways); industrial equipment (different types of engines, pumps, compressors, gasoline generators and machine-tools equipment).

Application area of the energy harvesters is supplying for low-power wireless sensors for online health and environmental monitoring, tracking devices (for instance, transport tracking), industrial sensors (temperature control and air quality control).

There are three main vibrational energy conversion approaches: induction method, electrostatic and piezoelectric method [2]. Amongst before mentioned, the piezoelectric method is the most promising one due to the greatest output electrical power density [3]. The main element of a vibrational piezoelectric transducer is a piezoelectric cantilever beam. The key feature of this structure is a steep increase in an efficiency of energy conversion at the resonant frequency of the beam.

\footnotetext{
* Corresponding author: ostsilograf@ya.ru
} 
There is some work, which demonstrates a possibility to generate electrical voltage of ones and tens volts and power of hundreds of microwatts when using a low-frequency $(<200$ $\mathrm{Hz}$ ) piezoelectric transducer [4-7].

AC magnetic field of $50 \mathrm{~Hz}$ is another prospective energy source. The main AC magnetic field sources in urban environments are electrical machinery (electrical engines, pumps, compressors, transformer stations) and lightning equipment (ballasts for fluorescent lamps).

The main technique of magnetic energy conversion is based on Faraday's law and usage of an electromagnetic coil. Faraday's law relates the rate of change of magnetic flux through a cross-section of the coil to the magnitude of the electro-motive force induced in the coil. However, this approach has a serious drawback: the lower a frequency of AC magnetic field the more turns the coil should contain to maintain the given level of output voltage, according to Faraday's law. It is for this reason that low-frequency vibrational electromagnetic transducers are bulky and expensive.

In this work, a method of AC magnetic field conversion, which combines advantages of a high-efficiency piezoelectric method, is proposed.

\section{Design and principle of action}

The main element of the proposed energy harvester is a vibrational transducer. The transducer represents a cantilever beam clamped at one end whereas another end is free (Fig.1). The beam consists of a piezoelectric element, a spring element and a magnetic mass.

A commercially available piezoelectric bimorph based on PZT-19 piezoceramics was used as the piezoelectric element. The bimorph with total dimension of $32 \times 12 \times 0.6 \mathrm{~mm}^{3}$ was glued at one end to a base of FR4 textolite with total dimension of $12 \times 12 \times 1.5 \mathrm{~mm}^{3}$. Output electrodes were deposited on the base. A stainless steel layer with dimensions of 45 $\times 12.5 \times 0.2 \mathrm{~mm}^{3}$ was used as the spring element. The spring element was glued to the piezoelectric bimorph by cyanoacrylate glue. The magnetic mass of $\mathrm{NdFeB}$ magnets with dimensions of $5 \times 5 \times 17 \mathrm{~mm}^{3}$, mass $m=1.1 \mathrm{~g}$ and remnant magnetic flux density $\mathrm{Br}=3.5$ $\mathrm{kOe}$ was mounted at the free end of the spring element. A magnetic moment of the mass was oriented perpendicularly to the spring element plane. The spring element and the mass were used to configure a resonant frequency of the transducer to $f_{0}=50 \mathrm{~Hz}$. Dimensions of the spring element and the tip mass were calculated by means of finite-element software COMSOL Multiphysics.

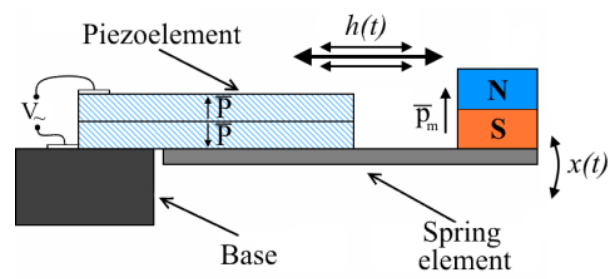

Figure 1. A structure of the vibrational transducer.

A principle of action of the transducer is as follows. Applying an external AC magnetic field $h(t)$ along the beam, magnetic moment of the mass aligns toward magnetic lines of $h(t)$ causing a periodic rotational movement of the mass. The magnetic torque $\mathbf{N}$ of a permanent magnet placed in an external magnetic field $\mathbf{h}$ is described as follows [8]:

$$
\mathbf{N}=\mu_{0} \cdot\left(\mathbf{p}_{\mathbf{m}} \times \mathbf{h}\right)=\mu_{0} \cdot V \cdot(\mathbf{M} \times \mathbf{h})
$$

Where $\mathbf{p}_{\mathbf{m}}$ and $\mathbf{M}$ are magnetic moment and magnetization of the permanent magnet, respectively; $\mu_{0}$ - magnetic permeability of vacuum; $V$ - volume of the permanent magnet. 
A periodical movement of the magnetic mass $x(t)$ leads to a deflection of the beam so that bending oscillations occur in the structure. The deflection of the beam causes mechanical stress in the piezoelectric element, which generates electrical voltage due to the piezoelectric effect.

Regarding Euler-Bernoulli beam theory, natural frequency $f_{b}$ of bending modes of the cantilever beam is proportional to the following parameters [9]:

$$
f_{b} \sim \frac{1}{L^{2}} \sqrt{\frac{E I}{A \rho}}
$$

Where $L, E, I, A, \rho$ are length, Young's modulus, second area moment, cross-section area and density of the beam, respectively.

Given that any electronics require a stabilized level of DC voltage as supply (for instance, $3.3 \mathrm{~V}$ ), the harvester should also contain an AC/DC-converter. In this work the converter is based on an LTC3588 chip. The chip consists of a low-loss full-wave integrated bridge rectifier and a DC/DC switching regulator. The converter works in the following way: when amplitude of input AC signal exceeds a particular threshold level, DC voltage of fixed level $(1.8 \mathrm{~V}, 2.5 \mathrm{~V}, 3.3 \mathrm{~V}$ or $3.6 \mathrm{~V})$ appears on the output. A model of an assembled harvester is shown on the Fig.2.

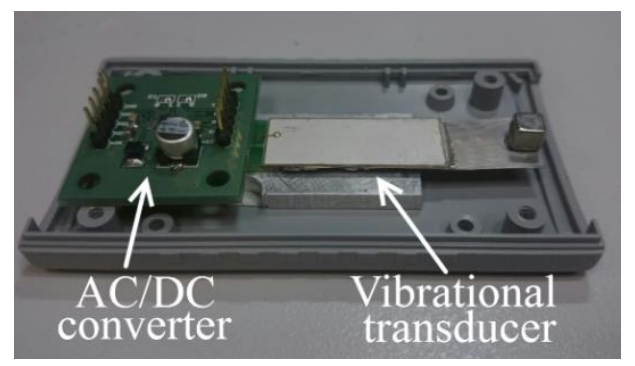

Figure 2. The assembled energy harvester.

\section{Experimental setup}

The measurement setup used for investigations is shown in Fig.3. The setup consists of a pair of Helmholtz coils, a function generator Agilent 33210A, a digital voltmeter AKIP-2401 and a personal computer (PC) with installed LabVIEW software. The transducer (1) is placed into an external AC magnetic field with a frequency in the range of $1-100 \mathrm{~Hz}$ and a resulting magnetic field amplitude in the range of $0.5-5$ Oe. Magnetic lines of the applied AC magnetic field are parallel to the plane of the transducer. Given position of the transducer provides the rotational movement of the magnetic tip mass so that bending oscillations occur in the beam. The AC magnetic field is induced by the Helmholtz coils (2), supplied by the functional generator (3). The output voltage from the transducer is measured by the digital voltmeter (4). The PC (5) provides control of the measuring equipment, data acquisition and processing. 


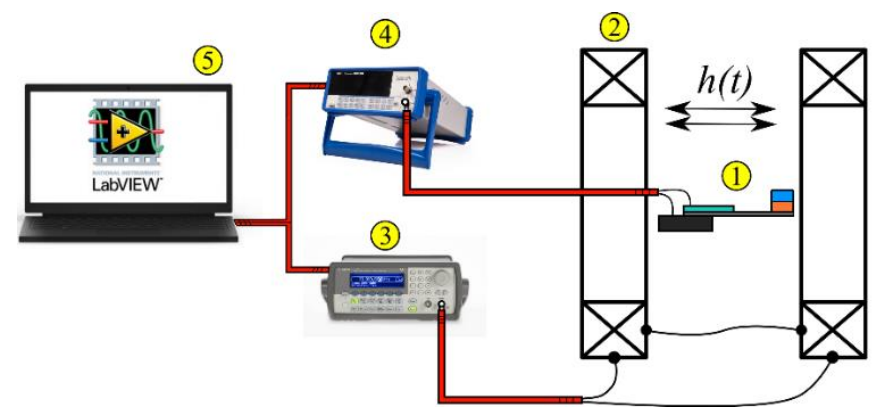

Figure 3. A setup for magnetic measurements of the transducer.

\section{Results and discussion}

For characterization of the energy harvester, it is necessary to investigate properties of both the vibrational transducer and the harvesting system as a whole. Frequency response investigations should be carried out to determine an operating point of the transducer, which corresponds to the resonant frequency $f_{0}$. Another important stage is dedicated to electrical power measurements. To characterize the electronic part of the harvester, charging time and time dependencies of DC output voltage should be investigated. The original resonant frequency of the transducer equals $f_{0}=50 \mathrm{~Hz}$ and corresponds to the frequency of industrial AC magnetic field.

The frequency response of the transducer measured at different amplitudes of AC magnetic field $h(t)$ is shown in Fig.4.

It can be seen that the curve is symmetrical at the amplitude $h=0.5$ Oe. However, a subsequent increase in the amplitude of magnetic field leads to a resonant frequency offset to a low-frequency domain. At the same time, asymmetry in curves is observed. With the increase in frequency from $40 \mathrm{~Hz}$ to $60 \mathrm{~Hz}$ and amplitudes $h>0.5$ Oe the amplitudefrequency curves show a steep rise, reach a peak at $f_{0}$ and gradually lower at frequencies $f>$ $f_{0}$. Curves at $h=3.75-5$ Oe are getting smoother on the left than at smaller fields. The observed behavior is characterized by a change in the stiffness of the vibrational transducer [10]. In our case, the oscillatory system is getting softer while increasing the amplitude of AC magnetic field.

The maximum value of output rms voltage is $U_{r m s}=12.6 \mathrm{~V}$ at the frequency $f=48 \mathrm{~Hz}$ and $U_{r m s}=5.6 \mathrm{~V}$ at the frequency $f=50 \mathrm{~Hz}$ at a constant $\mathrm{AC}$ field of $h=5 \mathrm{Oe}$. Investigations were provided at two resonant frequencies: initial $50 \mathrm{~Hz}$ and final $48 \mathrm{~Hz}$. This was due to the aforementioned resonant frequency offset determined by the amplitude of AC magnetic field.

A typical waveform of voltage generated by the transducer in the excitation field of frequency $f=48 \mathrm{~Hz}$ with amplitude $h=5$ Oe is shown in Fig.5.

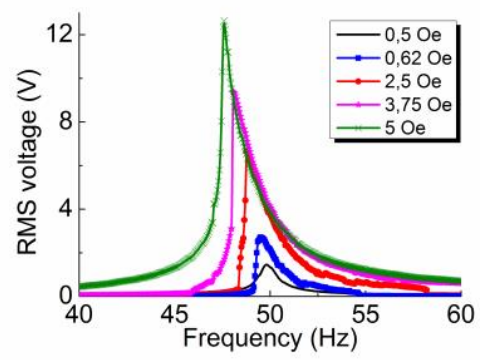

Figure 4. Frequency response of the transducer at different amplitudes of $h$. 


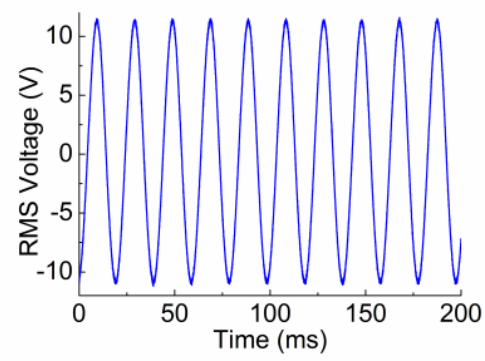

Figure 5. Time dependence of output electrical voltage at $h=5$ Oe and $f=48 \mathrm{~Hz}$.

It is shown that the signal represents a sinusoidal curve with a constant amplitude of $U_{r m s}=$ $12.6 \mathrm{~V}$. The signal has a low noise indicating a high work stability of the transducer.

Next step of investigations is dedicated to electrical power measurements. During this investigation, the transducer was placed into an external AC magnetic field $h(t)$ of the frequency $f_{0}$. The transducer was connected with a load resistance of different values and a voltage drop was measured across it. The measured voltage was recalculated into an electrical power. The load dependencies are presented at Fig.6. Due to the frequency offset the measurements were provided both at the initial $f=50 \mathrm{~Hz}$ and the final $f=48 \mathrm{~Hz}$ at constant amplitude of the AC field $h=5$ Oe. It can be seen that the maximal rms power reached a peak of $P_{r m s}=37 \mu \mathrm{W}$ at $f=50 \mathrm{~Hz}$ and $P_{r m s}=200 \mu \mathrm{W}$ at $f=48 \mathrm{~Hz}$. The maximum power was observed at a resistance of $R=330 \mathrm{kOhm}$. The maximum voltage across the load reached a peak of $U_{r m s}=5.4 \mathrm{~V}$ at $f=50 \mathrm{~Hz}$ and $U_{r m s}=12.6 \mathrm{~V}$ at $f=48 \mathrm{~Hz}$. The maximum voltage drop was observed at a resistance $R=10 \mathrm{MOhm}$.

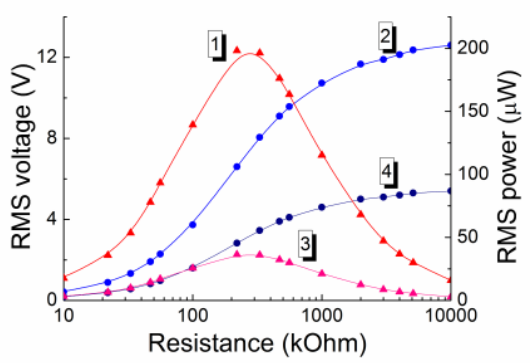

Figure 6. Output rms power and voltage of the transducer vs. load resistance: $1-$ output power at $f=$ $48 \mathrm{~Hz} ; 2$ - output voltage at $f=48 \mathrm{~Hz} ; 3$ - output power at $f=50 \mathrm{~Hz} ; 4$ - output voltage at $f=50 \mathrm{~Hz}$.

Charging time of the energy harvester is a very important parameter because it determines a turn-on delay of the device. This fact is particularly important if AC magnetic fields appear and disappear periodically (for instance, electric motor can turn on and turn off periodically). In this case, the harvester has to have enough time to be charged and supplying a connected load. However, in most modern papers only power dependencies are presented. This is insufficient to describe the harvesting system as a whole. Therefore, investigations of charging time and output voltage of the harvester are crucially important.

Time dependencies of output stabilized DC voltage of the harvester are presented in Fig.7. During this investigation the transducer was places into an external AC magnetic field $h(t)$ of the frequency $f=f_{0}=48 \mathrm{~Hz}$ and constant amplitude of $h=5$ Oe. The transducer was connected with AC/DC-converter. The stabilized voltage was measured at the output of the converter. 


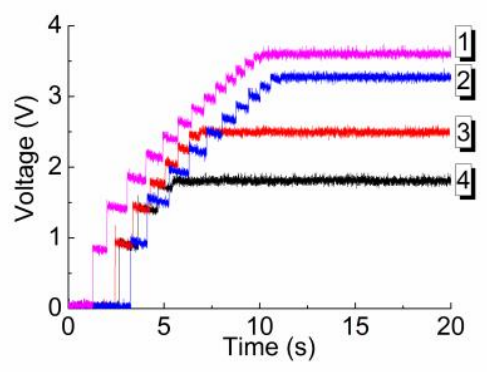

Figure 7. Time dependencies of stabilization of output voltage of the harvester: $1-1.8 \mathrm{~V}, 2-2.5 \mathrm{~V}$, $3-3.3 \mathrm{~V}, 4-3.6 \mathrm{~V}$.

Depending on preferences, the harvester can stabilize DC voltage of $1.8 \mathrm{~V}, 2.5 \mathrm{~V}, 3.3 \mathrm{~V}$ or $3.6 \mathrm{~V}$. It can be seen that output voltage is growing in steps up to corresponding output voltage level. Charging time depends on the voltage level and have a maximum value $t \approx 11$ $\mathrm{s}$ for $3.6 \mathrm{~V}$ and minimum value $t \approx 6 \mathrm{~s}$ for $1.8 \mathrm{~V}$.

\section{Conclusion}

In this paper, the energy-harvesting device based on a vibrational piezoelectric transducer for AC magnetic field conversion is presented. The maximal $\mathrm{rms}$ power of the transducer reached a peak of $P_{r m s}=37 \mu \mathrm{W}$ at $f=50 \mathrm{~Hz}$ and $P_{r m s}=200 \mu \mathrm{W}$ at $f=48 \mathrm{~Hz}$. If the harvester is placed into an external AC magnetic field $h(t)$ of the frequency $f=48 \mathrm{~Hz}$ and constant amplitude of $h=5 \mathrm{Oe}$, a regulated DC voltage of fixed level $(1.8 \mathrm{~V}, 2.5 \mathrm{~V}, 3.3 \mathrm{~V}$ or $3.6 \mathrm{~V})$ appears on the output of the device. Charging time depends on the voltage level and has the maximum value of $t \approx 11 \mathrm{~s}$ for $3.6 \mathrm{~V}$ and minimum value of $t \approx 6 \mathrm{~s}$ for $1.8 \mathrm{~V}$ output.

The Research was supported by the Ministry of Education and Science of Russia, project №8.1183.2017.

\section{References}

[1] M.-G. Kang, W.-S. Jung, C.-Y. Kang, S.-J. Yoon, Actuators, 5, 5 (2016)

[2] T. J. Kazmierski, S. Beeby (eds.), Energy Harvesting Systems, New York: Springer (2011)

[3] S. Roundy, P. K. Wright, J. Rabaey, Computer Communications, 26, 11, 1131-1144 (2003)

[4] S. Roundy, P. K. Wright, Smart Materials and Structures, 13, 5, 1131-1142 (2004)

[5] S. Roundy, E. S. Leland, J. Baker, E. Carleton, E. Reilly, E. Lai et al., IEEE Pervasive Computing, 4, 1, 28-36 (2005)

[6] E. S. Leland, P. K. Wright, Smart Materials and Structures, 15, 1413-1420 (2006)

[7] F. A. Fedulov, L. Yu. Fetisov, D. V. Chashin, Nano- i Mikrosistemnaya Tehnika, 19, 2, 114-121 (2017)

[8] J. D. Jackson, Classical Electrodynamics (New York: John Wiley \& Sons, 1998)

[9] R. F.Gibson, Principles of composite materials (New York: CRC Press, 2007)

[10]Z. Wang, S. Matova, R. Elfrink, M. Jambunathan, C. De Nooijer, R. Van Schaijk, R. J. M. Vullers, Trans. On 25th International Conference on Micro Electro Mechanical Systems (MEMS) “IEEE MEMS 2012”, 1201-1204 (2012) 